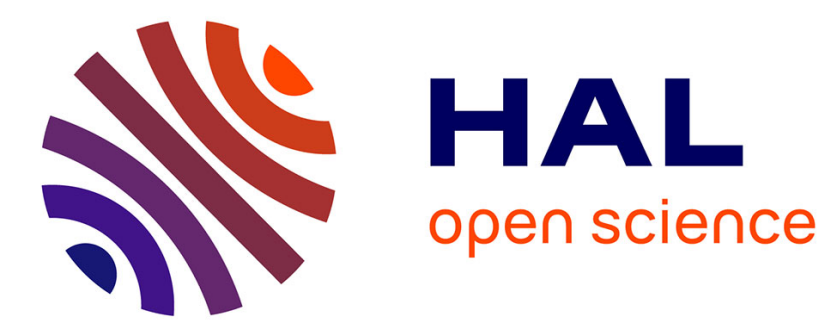

\title{
Impact of a knee orthosis over walking
}

Olivier Bordron, Clément Huneau, Eric Le Carpentier, Yannick Aoustin

\section{To cite this version:}

Olivier Bordron, Clément Huneau, Eric Le Carpentier, Yannick Aoustin. Impact of a knee orthosis over walking. ROMANSY 22 - Robot Design, Dynamics and Control. CISM International Centre for Mechanical Sciences (Courses and Lectures), vol 584. Springer, Cham, 2018, 978-3-319-78962-0. hal-01985287

\section{HAL Id: hal-01985287 \\ https://hal.science/hal-01985287}

Submitted on 17 Jan 2019

HAL is a multi-disciplinary open access archive for the deposit and dissemination of scientific research documents, whether they are published or not. The documents may come from teaching and research institutions in France or abroad, or from public or private research centers.
L'archive ouverte pluridisciplinaire HAL, est destinée au dépôt et à la diffusion de documents scientifiques de niveau recherche, publiés ou non, émanant des établissements d'enseignement et de recherche français ou étrangers, des laboratoires publics ou privés. 


\title{
Impact of a knee orthosis over walking
}

\author{
Olivier Bordron, Clément Huneau, Éric Le Carpentier, and Yannick Aoustin \\ University of Nantes, Centrale Nantes, LS2N, UMR CNRS 6004, Nantes, France \\ olivier.bordron@ls2n.fr
}

\begin{abstract}
This paper proposes a mathematical model for a 7-links anthropomorphic biped - composed with two identical legs, two feet and one trunk - with an orthosis attached to both thigh and calf, during walking. We consider a cyclic walking gait in the sagittal plane. In the first part, we obtain trajectories of the motion for different walking speeds. It confirmed the fact that much more torque is recquired in the stance leg than in the swing leg. The second part analyses the impact of an orthosis, which fully assists the knee over the walking. Simulations point out the fact that valid joints have to compensate the weight of the orthotic system and show the interest to assist the knee of the stance leg.
\end{abstract}

Keywords: biped, walking optimisation, orthosis, assistance.

\section{Introduction}

Exoskeletons appeared in the 1980s, for military applications [5]. Nowadays, we can see them in industry environment or medical field. They allow an operator to assist him in his motions or augment his strength. Active orthosis - simplified exeskeletons assisting in general one joint - have been developped in medical environment. They can be used for reducing chronic strains, compensating muscle impairments in case of rehabilitation or helping elderly people during walking. RobotKnee, a knee brace coupled with a linear actuator attached to both calf and thigh, provides a torque calculated from the measure of the vertical ground reaction force [9]. The orthosis EICoSI uses electromyograms to estimate the trajectory [7]. In [10], authors described a quasi-passive orthosis using spring assistance of the knee during contact with the ground, which is detected by an instrumented shoe.

Each assistance system has its own design, with its geometrical and mechanical properties. These features have an impact over walking and over the operator. A state of the art over lower limb exoskeletons [6] points out the fact that wearing walking assist devices has an influence over the metabolic cost, for instance the mean oxygen consumption. Then, to estimate the impacts of an orthosis over walking gait before designing the system is important.

Several studies using simulation and walking optimisation have already been done. For instance, [1] studied the better way to actuate a wearable walking assist device. In [11], walking gait optimisation is made over a 3D biped model. At 


\begin{tabular}{|l|c|c|c|c|}
\hline & Foot & Calf & Thigh & $\begin{array}{c}\text { Trunk (with } \\
\text { Head and Arms) }\end{array}$ \\
\hline Segment Weight (kg) & $m_{\mathrm{f}}=1.09$ & $m_{\mathrm{c}}=3.49$ & $m_{\mathrm{t}}=7.5$ & $m_{\mathrm{T}}=50.9$ \\
Segment Length $(\mathrm{m})$ & $L_{\mathrm{p}}=0.06$ & $L_{\mathrm{c}}=0.44$ & $L_{\mathrm{t}}=0.44$ & $L_{\mathrm{T}}=0.85$ \\
& $L_{\mathrm{d}}=0.21$ & & & \\
& $H_{\mathrm{f}}=0.07$ & & & \\
Inertia $\left(k g . \mathrm{m}^{2}\right)$ & $I_{\mathrm{f}}=0.02$ & $I_{\mathrm{c}}=0.17$ & $I_{\mathrm{t}}=0.36$ & $I_{\mathrm{T}}=8.95$ \\
Center of mass $(\mathrm{m})$ & $s p_{\mathrm{x}}=0.07$ & $s_{\mathrm{c}}=0.19$ & $s_{\mathrm{t}}=0.19$ & $s_{\mathrm{T}}=0.32$ \\
& $s p_{\mathrm{y}}=0.035$ & & & \\
\hline
\end{tabular}

Table 1. Anthropomorphic parameters of the human body.

last, [2] showed theoretically the feasibility and efficiency of a passive exoskeleton for human carrying a load.

Nevertheless, walking gait optimisation has never been done for assessing the cost of wearing an orthosis. We propose to integrate several properties of the orthosis in order to measure its impact over the human body during walking. As we simply want to highlight the advantages and drawbacks of wearing a knee orthosis, the study is carried out with a 2D planar model. The step of the walking is defined in the sagittal plane by a single support phase followed by an impact of the swing foot with the ground. Then, the dynamic model is completed with an orthosis either on the left or right knee. In both cases, the torque costs with and without orthosis are compared to carry out the same walking.

\section{Modelisation of the biped without assistive device}

In this part, we propose a dynamic model for the planed biped during walking.

\subsection{Anthropomorphic parameters of the biped}

The model used to simulate a step is illustrated Figure 1. This model is composed of one trunk, two legs - each one is composed with both a thigh and calf - and two feet. Model parameters of each link, such as inertia, mass, length and position of the center of mass, are chosen by using Winter's anthropomorphic data (see Table 1) [12].

\subsection{Dynamic model}

Considering the left foot flat on the ground (the left foot forms a complete joint with the ground), that is to say for any time $q_{\mathrm{p}_{1}}=0$ (see Figure 1), and taking the origin of the reference frame at the left ankle, the created dynamic model of the seven-link biped is:

$$
\mathbf{D}(\mathbf{q}) \ddot{\mathbf{q}}+\mathbf{C}(\mathbf{q}, \dot{\mathbf{q}}) \dot{\mathbf{q}}+\mathbf{G}(\mathbf{q})=\mathbf{B} \boldsymbol{\Gamma}+\mathbf{J}^{\top} \mathbf{F}
$$



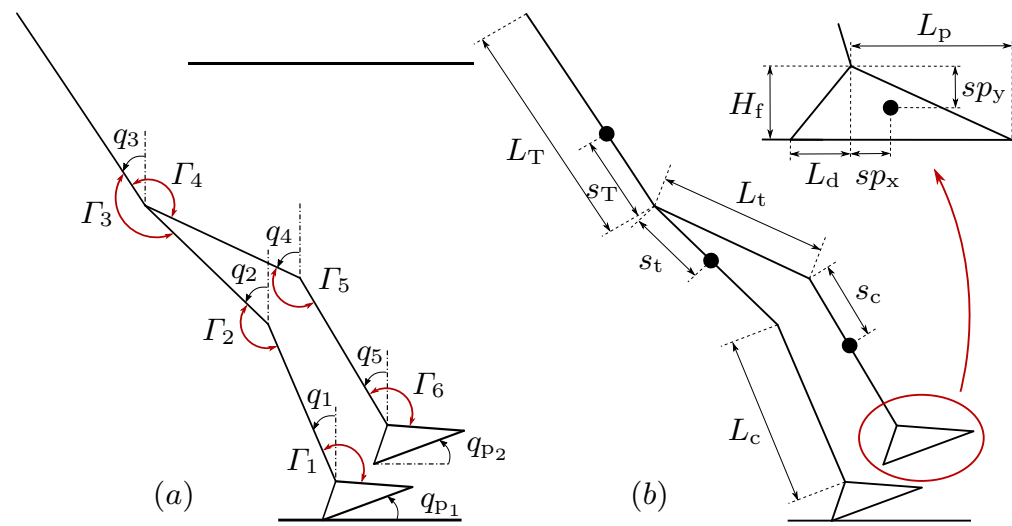

Fig. 1. Modelisation of the planed biped. a) Parameterisation of the biped. Note that angles are positive in counterclockwise. b) Length segments and position of the centers of mass.

where $\mathbf{D}$ is the $6 \times 6$ inertia matrix, $\mathbf{C}$ is the $6 \times 6$ matrix representing Coriolis effects and $\mathbf{G}$ is the $6 \times 1$ gravity matrix. The $6 \times 1$ vector $\mathbf{q}=\left(\begin{array}{lllll}q_{1} & q_{2} & q_{3} & q_{4} & q_{5} \\ \mathrm{p}_{2}\end{array}\right)^{\top}$ contains joint angles represented in Figure 1 and $\boldsymbol{\Gamma}=\left(\Gamma_{1} \Gamma_{2} \Gamma_{3} \Gamma_{4} \Gamma_{5} \Gamma_{6}\right)^{\top}$ is the $6 \times 1$ torque vector.

$\mathbf{B}$ is a constant matrix, composed of zeros and units, to take into account of the torques on the joints. J, a Jacobian matrix obtained with virtual works, can be obtained by using conditions of flat contact of the stance foot 1 with the ground.

\subsection{Geometric and kinematic conditions}

The walking gait studied here is cyclic. Thus the walking gait can be defined by designing over one step only. This step is defined by a phase of single support and an impact with the ground. The impact model is the same used in [3]. We assume that trajectories describing the motion are defined by polynomial functions of fourth order, depending on time. Consequently, for each joint, the trajectory can be determined with five geometric or kinematic conditions. We use initial and final positions (respectively $\mathbf{q}_{0}$ and $\mathbf{q}_{\mathbf{f}}$ ), initial and final velocities (respectively $\dot{\mathbf{q}}_{0}$ and $\dot{\mathbf{q}}_{\mathrm{f}}$ ) and the position at mid-cycle $\mathbf{q}_{\mathrm{int}}$. Thanks to the cyclic conditions, we link the final conditions to the initial conditions in position and velocity (see Figure 2). Moreover, at initial and final positions, characterised by both feet flat on the ground, the configuration of the legs are totally determined by the configuration of the hip $\left(x_{\mathrm{h}}, y_{\mathrm{h}}\right)$ and the step length $d$. Thus, the entire cyclic gait can be described with these parameters:

$$
\left(x_{\mathrm{h}_{\mathrm{f}}}, y_{\mathrm{h}_{\mathrm{f}}}, d, q_{3_{\mathrm{f}}}, \dot{\mathbf{q}}_{\mathrm{f}}, \mathbf{q}_{\mathrm{int}}\right)
$$

The design of the walking gait is made under the constraints: no take off and no 


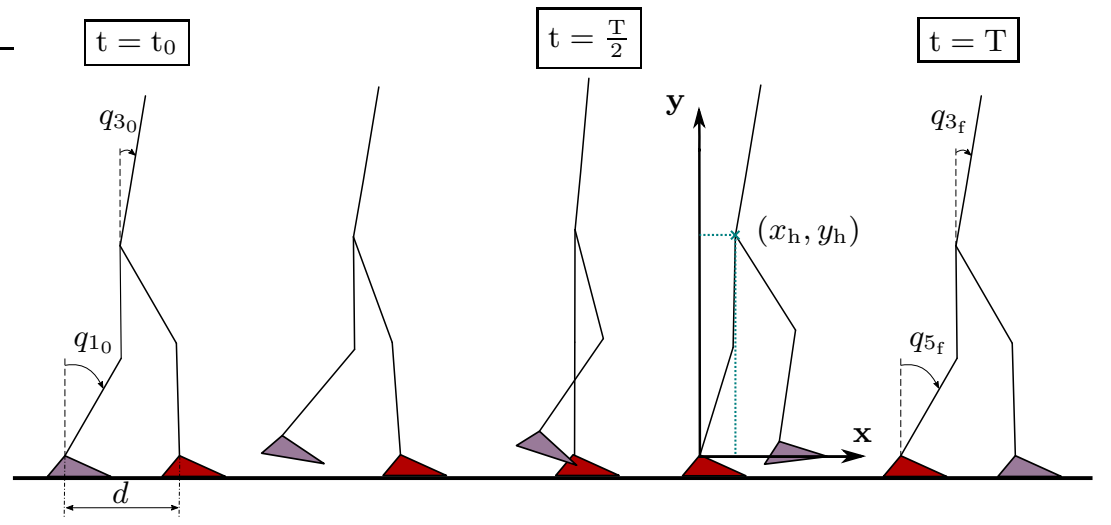

Fig. 2. A step of the biped and conditions of cyclicality.

sliding of the stance foot during the single support, the center of pressure must be inside the sole surface of the foot on the ground, and limits in magnitude of the joint torques and joint velocities.

\section{Optimal trajectories of the planed biped}

\subsection{Optimisation}

The cost function used for optimisation is the sthenic criterion [4]: the optimal trajectories minimise joint torques produced by the human body. From a data set of (2), positions, velocities and acceleration designing the walking cycle are determined (see Section 2.3). With the dynamic model (1) of the biped, joints torques then the sthenic criterion can be determined.

The optimisation is done by a Sequential Quadratic Programming (SQP) method (quasi-Newton method [8] implemented in the Matlab function fmincon).

For different walking speeds (from 0.2 to $1.4 \mathrm{~m} . \mathrm{s}^{-1}$, by increment of 0.1 ), we optimise the vector $\left(x_{\mathrm{h}_{\mathrm{f}}}, y_{\mathrm{h}_{\mathrm{f}}}, d, q_{3_{\mathrm{f}}}, \dot{\mathbf{q}}_{f}, \mathbf{q}_{\text {int }}\right)$. The cost and the step length $d$ obtained by optimisation are given in Figure 3. Because this optimisation algorithm is very sensitive to initial conditions, we use the set of optimised parameters obtained at walking speed $v-0.1$ as the initial set of parameters for walking speed $v$. It corresponds to blue circle in Figure 3.

A second series of optimisation is done with the same principle except we update walking speed with decrement of $0.1 \mathrm{~m} . \mathrm{s}^{-1}$ (plus orange symbol in Figure 3). At last, for each walking speed, we optimise once more by using, as initial conditions, the set of optimised parameters obtained in the second series of optimisation (cross violet symbol). We notice that both cost and step length increase with walking speed. This feature is consistent with our natural walking gait. The results of the last series of optimisation will be used in the next section. 

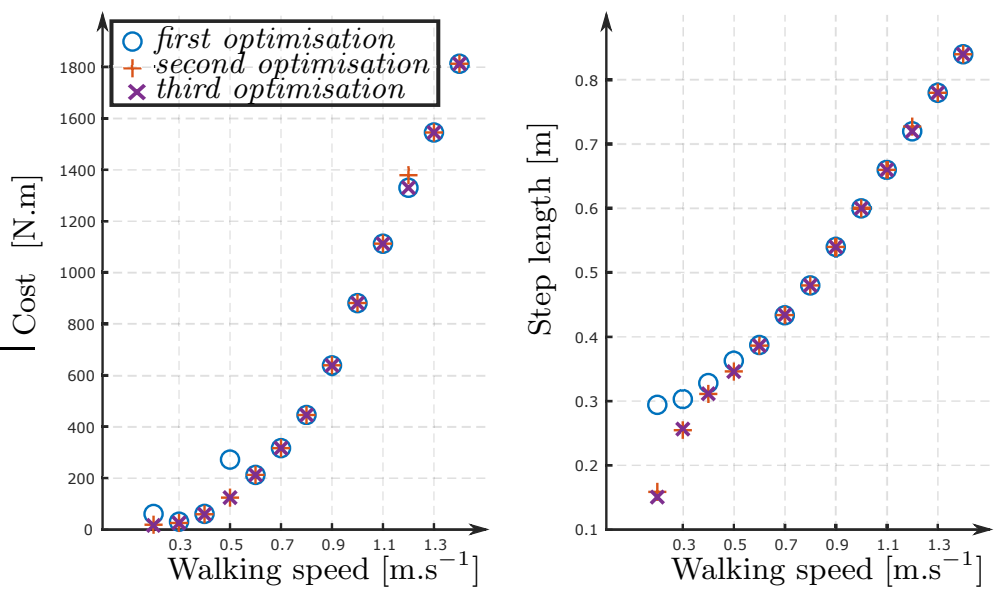

Fig. 3. Cost and step length for different walking steps.

\begin{tabular}{|l|c|c|}
\hline & Calf & Thigh \\
\hline Segment Weight $(\mathrm{kg})$ & $m_{\mathrm{c}_{\mathrm{orth}}}=1.0$ & $m_{\mathrm{t}_{\mathrm{orth}}}=1.0$ \\
Inertia $\left(\mathrm{kg} . \mathrm{m}^{2}\right)$ & $I_{\mathrm{c}_{\mathrm{orth}}}=0.0367$ & $I_{\mathrm{t}_{\mathrm{orth}}}=0.0626$ \\
\hline
\end{tabular}

Table 2. Features of the orthosis.

\section{Presentation of the orthosis}

We add an orthosis to the biped model (see Figure 4). It is modeled by one additional mass with inertia to both calf and thigh. Its features are given in Table 2. Fot the sake of simplicity, we assume that the center of mass position of both calf and thigh are not affected by the orthosis.

We assume that the orthosis is placed on the left knee (knee of the support leg). Dynamic model of (1) changed for:

$$
\tilde{\mathbf{D}}(\mathbf{q}) \ddot{\mathbf{q}}+\tilde{\mathbf{C}}(\mathbf{q}, \dot{\mathbf{q}}) \dot{\mathbf{q}}+\tilde{\mathbf{G}}(\mathbf{q})=\mathbf{B}_{\text {hum }} \boldsymbol{\Gamma}_{\text {hum }}+\mathbf{B}_{\text {orth }} \Gamma_{\text {orth }}+\mathbf{J}^{\top} \mathbf{F}
$$

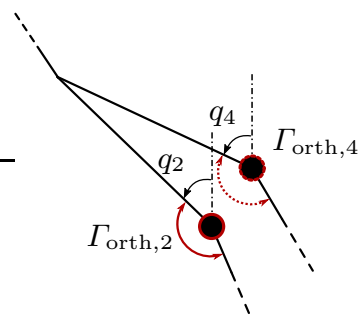

Fig. 4. Modelisation of the planed biped with orthosis. Because we want to study the effect of an orthosis on the whole walking cycle, its position can change (dotted circle). 

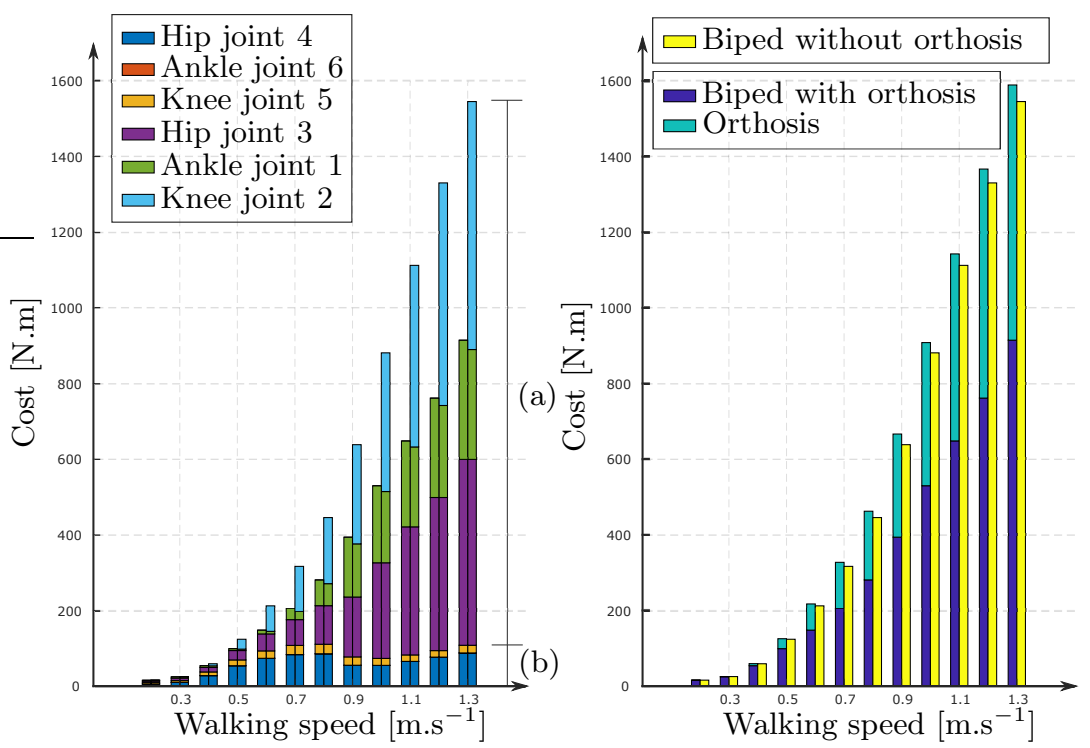

Fig. 5. Cost spent by the biped without orthosis and cost spent by the biped with orthosis (in case of orthosis positioned on the knee of the stance leg). For each walking speed, left bar corresponds to the biped with orthosis and right bar to the biped without orthosis. Note that: (a) Cost of the stance leg, (b) Cost of the swing leg.

where $\mathbf{B}_{\text {hum }}=\mathbf{B}$ (see eq. 1) and $\mathbf{B}_{\text {orth }}=\left(\begin{array}{llllll}-1 & 1 & 0 & 0 & 0 & 0\end{array}\right)^{\top}$.

\subsection{Influence of the orthosis over the joint torques}

In the previous section, we have calculated walking trajectories for different walking speeds in accordance with the cost function $J$. Now, we will use these optimal trajectories and suppose that the orthosis fully assists the knee. This situation allows us to evaluate the maximum benefit of the orthosis. In order to understand the effect of the orthosis over the whole step we are considering two cases:

- Orthosis positioned on the knee of the stance leg (Figure 5). Here, only the left knee and ankle are supporting the weight of the orthosis. Consequently, the cost of the biped ankle is higher with assistance than without.

- Orthosis positioned on the knee of the swing leg (Figure 6). In that case, all the joints - except ankle of the swing leg - feel the weight of the orthosis. Compared to the other case, the orthosis needs to produce less torque whereas the other joints have to produce more torque in order to compensate the assistance's weight. That is why the cost of the biped with orthosis is higher than the cost of the biped without. 

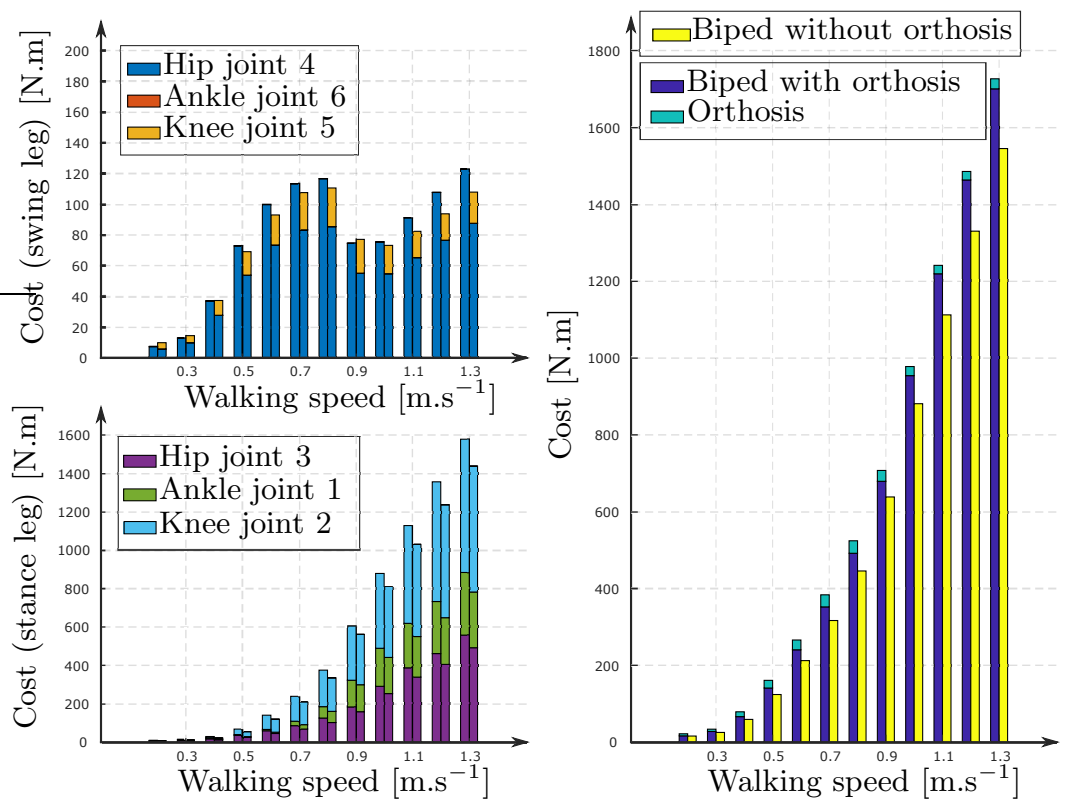

Fig. 6. Cost spent by biped without orthosis and cost spent by biped with orthosis (in case of orthosis positioned on the knee of the swing leg). For each walking speed, left bar corresponds to the biped with assistance and right bar to the biped without assistance.

\section{Conclusion}

This paper has compared the walking torque cost of a seven-links biped with and without orthosis for the same walking gait. Firstly, simulations confirmed that, in a single support phase, the addition of an orthosis increases the total torque cost during the walk. Then, they showed that it is much more interesting to assist the knee when the latter is a part of the stance leg. For instance, at walking speed $0.7 \mathrm{~m} . \mathrm{s}^{-1}$, when the knee is fully assisted, the orthosis contributes to nearly $37 \%$ of the total torque cost of the walking step if the latter belongs to the stance leg, against $8 \%$ if it belongs to the swing leg. Finally, it has been shown that, when the orthosis is part of the leg touching the ground, the biped reduces its total torque cost. Nevertheless, when the orthosis is part of the swing leg, the biped increases its total torque cost because it has to support the orthosis's weight.

The obtained results are based on a simple planar model of the human, who is equipped with an orthosis. For the study of a walk, a 2D model can give a general trend, which is not generally contradicted by the use of a more complex 3D model, even if the provided information is richer. And the weight of the orthosis is a handicap, which can be well taken into account with a planar motion. This numerical study has to be extended with an experimental data with a orthosis 
prototype. Furthermore, from this study, a possible extension is to know how to assist the knee in optimal manner by taking into account the motorisation's constraints.

\section{References}

1. Aoustin, Y., Chevallereau, C., Arakelyan, V.: Study and Choice of Actuation for a Walking Assist Device. In: H. Bleuler, M. Bouri, F. Mondada, D. Pisla, A. Rodic, P. Helmer (eds.) New Trends in Medical and Service Robots, pp. 3-12. Springer International Publishing, Cham (2016)

2. Aoustin, Y., Formalskii, A.M.: Strategy to Lock the Knee of Exoskeleton Stance Leg: Study in the Framework of Ballistic Walking Model. In: P. Wenger, C. Chevallereau, D. Pisla, H. Bleuler, A. Rodić (eds.) New Trends in Medical and Service Robots, pp. 179-195. Springer International Publishing, Cham (2016)

3. Aoustin, Y., Formalskii, A.M.: Walking of biped with passive exoskeleton: Evaluation of energy consumption. Multibody System Dynamics pp. 1-26 (2017). DOI 10.1007/s11044-017-9602-7

4. Chevallereau, C., Bessonnet, G., Abba, G., Aoustin, Y. (eds.): Walking Pattern Generators. ISTE (2009). URL http://onlinelibrary.wiley.com/doi/10.1002/ 9780470611623.ch4/summary. DOI: 10.1002/9780470611623.ch4

5. Dollar, A.M., Herr, H.: Lower Extremity Exoskeletons and Active Orthoses: Challenges and State-of-the-Art. IEEE Trans. on Robotics 24(1), 144-158 (2008). DOI 10.1109/TRO.2008.915453

6. Huo, W., Mohammed, S., Moreno, J.C., Amirat, Y.: Lower Limb Wearable Robots for Assistance and Rehabilitation: A State of the Art. IEEE Systems Journal 10(3), 1068-1081 (2016). DOI 10.1109/JSYST.2014.2351491

7. Mefoued, S.: A second order sliding mode control and a neural network to drive a knee joint actuated orthosis. Neurocomputing 155, 71-79 (2015). DOI 10.1016/j. neucom.2014.12.047

8. Nocedal, J., Wright, S.: Numerical Optimization. Springer Science \& Business Media (2006)

9. SCHMITT, C.: A study of a knee extension controlled by a closed loop functional electrical stimulation. Proc. 9th Annu. Conf. of the Int. FES Society, Bournemouth, 2004 pp. 135-137 (2004). URL https://ci.nii.ac.jp/naid/20000716730/en/

10. Shamaei, K., Cenciarini, M., Adams, A.A., Gregorczyk, K.N., Schiffman, J.M., Dollar, A.M.: Design and evaluation of a quasi-passive knee exoskeleton for investigation of motor adaptation in lower extremity joints. IEEE Trans. on biomedical engineering 61(6), 18091821 (2014). DOI 10.1109/tbme.2014.2307698. URL https://doi.org/10.1109/TBME.2014.2307698

11. Tlalolini, D., Aoustin, Y., Chevallereau, C.: Design of a walking cyclic gait with single support phases and impacts for the locomotor system of a thirteen-link $3 \mathrm{~d}$ biped using the parametric optimization. Multibody System Dynamics 23(1), 33 (2009). DOI 10.1007/s11044-009-9175-1. URL https://doi.org/10.1007/s11044-009-9175-1

12. Winter, D.A.: Biomechanics and Motor Control of Human Movement. John Wiley \& Sons (2009) 